\title{
Image Analysis using Deep Learning Techniques
}

\author{
P. Jayasri, N. Subhash Chandra
}

\begin{abstract}
In late years, critical learning methodologies especially Convolutional Neural Networks have been utilized in different solicitations. CNN's have appeared to be a key capacity to ordinarily expel broad volumes of data from massive information. The uses of CNNs have inside and out ended up being useful especially in orchestrating ordinary pictures. Regardless, there have been essential obstacles in executing the CNNs in a restorative zone as a result of the nonattendance of genuine getting ready data. Consequently, general imaging benchmarks, for instance, Image Net have been conspicuously used in the restorative not too zone notwithstanding the way that they are perfect when appeared differently about the CNNs. In this paper, a comparative examination of LeNet, AlexNet, and GoogLeNet has been done. Starting there, the paper has proposed an improved hypothetical structure for requesting helpful life structures pictures using CNNs. In perspective on the proposed structure of the framework, the CNNs building are required to beat the previous three plans in requesting remedial pictures.
\end{abstract}

Index Terms: Convolutional Neural Networks, ImageNet, AlexNet, and GoogLeNet.

\section{INTRODUCTION}

There is a huge amount of pictures that are taken in centers once every day [1]. Along these lines, there is a critical augmentation in weight on the social protection providers for them to give exact and powerful symptomatic organizations. As demonstrated by [2], the National Institute of Medicine has assessed that generally more than 15 million patients in America are wrongly broke down on a yearly reason. This is a critical massive number of people who are being misdiagnosed and it is a vital issue if these returns. This issue is oozing from the route that there are significant volumes of imaging data made each day in the medicinal centers and there are no proper systems set up that can be used to correctly and capably handle such kind of data [1]. In like manner, there is a prerequisite for progressively careful and shrewd decision genuinely strong systems for masters with the objective that they can on a very basic level abatement the number of patients who are misdiagnosed [3]. As shown by [4], significant learning is a development that is spurred from the working of human personalities. The frameworks of phony neurons can take a gander at gigantic proportions of data to thusly see fundamental models without the prerequisite for individuals to be available [5]. So to speak, counterfeit neural frameworks work thusly as humans do. Nevertheless, since they are structures, the phony neural frameworks perform better when appeared differently with people. A great part of

Revised Manuscript Received on 14 August, 2019.

P. Jayasri, PG Scholar in CSE, CVRCE, Hyderabad, Telangana, India(Email: jayasripayili@gmail.com)

Dr.N. Subhash Chandra, Professor in CSE, CVRCE, Hyderabad, Telangana, India(Email: subhashchandra.n.cse@gmail.com) the time, significant learning is essentially used where there is a need for requesting structures in undefined data [6]. Thusly significant learning is generally used to perceive unstructured models in various media, for instance, content, sound, video and helpful pictures [7]. Exactly when the pros are taking a gander at the patients, they endeavor reliably to deal with refined issues. All things considered, the target of various restorative pros is to give the correct treatment to the patients subject to the open past remedial reports like the lab test reports, signs and signs of the patients, therapeutic pictures similarly as the patient's restorative history [8]. An examination driven by [9] demonstrated that the electronic restorative administration's data is evaluated to grow distinctly from 500 petabytes to 25,000 petabytes by 2020 all through the whole world. As noted before, it is a noteworthy test for remedial experts to get definite understandings from billions of clinical data. Along these lines, endless, therapeutic specialists and data analysts are unendingly finding answers for improvement calm thought in the focuses and crisis facilities. In this present day of improved development, there is a need to execute significant learning in a helpful business. As shown by [7], AI figuring's can do information dealing with and model affirmation and recognizing verification in an unrivaled way when stood out from individuals. Besides, AI estimations can be used to handle chance parts for diseases in incredibly colossal people. Besides, the AI figuring's can in like manner be used to perceive and envision perilous ailments, for instance, illness, diabetes, etc. As demonstrated by [8], the use of PC helped to discover (CAD) to overview compasses of women can distinguish around 52\% of the threat before the women were investigated officially. "Reference [10]" saw that AI counts can be used in various controls of medicine including exposure drugs, fundamental administration in clinical. Furthermore, the use of AI computations can change by a colossal edge the way by which medicine is penetrated to date. The power of AI estimations starting late has come when the remedial records are being digitized. Not at all like previously. Right when therapeutic records were essentially paper-based, these days, most remedial records are being secured electronically. Computer-based intelligence figuring can't work with paperbased therapeutic records. They can work if the remedial records are digitized. This infers these AI estimations has come at the ideal time when the restorative records are by and by being digitized. According to the use of electronic prosperity records (EHR) starting late has extended distinctly from generally $12 \%$ to $40 \%$ in the USA from 2007 
to 2012. Despite how therapeutic pictures are a basic piece of any patient's EHR, they are starting at now being poor down physically by human radiologists [11]. Individuals can't be stood out from machines since they are slower, they get exhausted and they most likely won't have much understanding. All these are the noteworthy confinements of using individuals when they appeared differently with AI counts. According to, an examination that is conceded and wrong can be dangerous to patients. In this way, it is crucial to motorize restorative picture examination utilizing definite and feasible AI counts. When daily, there is an extension in the number of restorative pictures, for instance, CT, MRI, and X-pillar. These kind of therapeutic pictures which are extending once multi-day are imperative in light of the way that they give principal information to experts to give exact assurance, helpful prescriptions, preparing similarly as giving remedial research [12]. With everything taken into account, the standard procedures used to recoup therapeutic pictures rely upon the remark of watchwords. In any case, contingent upon pictures remark isn't profitable because the method takes a huge amount of time and moreover it is difficult to depict the substance of these photos with words [13]. Beginning late, the has incredibly gotten qualification in the vocations of accommodating picture recovery and game-plan because of the improvement of getting ready to power and the unexpected degrees of progress in science and advancement correspondingly as broadband web. Similarly, the CBIR has likewise been related to remedial applications beginning late.

\section{RELATED WORK}

Various low dimension includes descriptors have been proposed as a picture portrayal extending from worldwide highlights, for example, shape and surface highlights as revealed in for an order of pneumonic knobs in lung CT pictures, edge highlights to the as of late utilized neighborhood highlight portrayals. Then again profound learning has indicated promising outcomes in picture arrangement. Profound learning implies a classification of AI methods, where various layers of data handling stages in progressive models are misused for example grouping and highlight learning.

To get the digital information more effectively there is the use of CNN (Convolution Neural Network) which is again as back propagation neural network. By considering multiple advantages of CNN (Convolution Neural Network), the future almost applications are implemented using CNN for better efficiency.

The CNN model can work for a huge amount of database with approximate 1000 classes as mentioned with very low FAR (False Acceptance Ratio). Mostly for feasible applications, there is a need for modifications in provided CNN (Convolution Neural Network). There are multiple convolution layers and pooling layers that help to connect the layers and check the performance by a combination of both pooling and convolution layers. One layer known as the convolution layer takes the much burden of work to compare to the pooling layer. The pooling layer improves the data rate by yielding the information available with a convolution neural network.
The weight updating is done by using the CNN layers mostly used layer is convolution layer. Most of the applications in biomedical such as brain tumor detection and classification, lung cancer detection also kidney stone detection uses CNN which is more effective in the medical domain. Information and analysis done by some users using CNN say that this Deep type of neural network also performs better in undesirable conditions.

Be that as it may, as far as research for ordering life structures in restorative pictures, there are just a couple of studies that have been completed utilizing CNN. One of the disadvantages of these examinations is that they don't give a broad assessment of achievement profound nets and are simply centered on single methodology, for example, just CT pictures were utilized in. To conquer this impediment, a design that can be summed up to different life systems with various modalities is required which prompts the primary focal point of this examination.

\section{MACHINE LEARNING ARCHITECTURE}

\section{Supervised Learning Models}

\section{Convolutional Neural Networks:}

At present, CNNs are the most researched AI counts in remedial picture assessment [14]. The clarification behind this is CNN's shield spatial associations while changing information pictures. As referenced, spatial affiliations are fundamental significance in radiology, for instance, a bone gets together with tissues, or where typical with hurtful tissue. An information picture of rough pictures, as well as changes it through Rectified Linear Unit (RELU) Layers as well as Convolutional Layers, what's more, Pooling Layers. This designates class scores or probabilities, hence requesting the commitment to the class with the most amazing probability.

\section{A. Convolution Layer:}

A convolution is an undertaking on two limits. In picture assessment, one limit involves data regards (for instance pixel regards) at a circumstance in the image, and the subsequent limit is lter each can be addressed as a bunch of numbers. Figuring the spot thing between the two limits gives a yield. The lter is then moved to the accompanying situation in the image as denied by the walk length. The count is reiterated until the entire picture is verified, making a component (or order) map. This is a guide of where the lter is determinedly activated and 'sees' apart, for instance, a straight line, a bit, or a bowed edge. In case a photograph of a face was supported into a $\mathrm{CNN}$, from the start low-level features, for instance, lines and edges are found by the lter. These advancements to constantly higher features in resulting layers, for instance, eyes, nose or ears, as the component maps become commitments for the accompanying layer in the $\mathrm{CNN}$ plan. 


\section{B. Pooling Layer:}

The Convolution, as well as RELU in a Pooling layer, is implanted between parameters to be resolved, similarly as the proportion of the image. Max-pooling is most conventionally used; other pooling layers fuse Average pooling and L2-institutionalization pooling. Max-pooling takes the greatest data regard inside alter and discards various characteristics; feasibly it diagrams the most grounded activations over a territory. The premise is that the general territory of an unequivocally instituted feature to another could undoubtedly contrast with its exact region.

\section{C: Fully Connected Layer:}

The nal layer in a CNN is the Fully Connected Layer, suggesting that every neural in the previous layer is related to every neuron. Related to the RELU, convolution as well as merge layers, that can be in any event 1 related layer depending upon the component of feature pondering needed. This layer takes the yield from the previous layer (RELU and Pooling, Convolutional) in information, as well as registers for request on the presence of other classes. On a very basic level, this layer looks blend of the most solidly sanctioned features that would show the image has a spot with a particular class. For instance, in slide histology glass, dangerous development cells extent diverged from standard cells. In case features of DNA were unequivocally recognized from the past layer, $\mathrm{CNN}$ would undoubtedly anticipate the closeness of danger cells. Standard neural framework getting ready methodologies with back spread [10] and stochastic edge drop help the CNN take in a basic relationship from planning pictures.

\section{PROPOSED METHODOLOGY}

The anatomical gathering issue is a fundamental development in Computer-Aided and Diagnosis Systems (CADs). The modify in Anatomical structures of fundamentally at present; CNNs are the most looked into AI calculations in therapeutic picture investigation. The explanation behind this is CNN's save spatial connections when sifting info pictures. As referenced, spatial connections are of critical significance in radial, the instance, it gets together with tissues. These are corn into a last Fully Connected Layer in doles outclass probabilities as well as scores, in this manner arranging the contribution to the class with the most elevated likelihood. Our proposed model of the CNN configuration is a modification of the fundamental designing of AlexNet [15]. This plan contains two convolutional layers (Conv) trailed by two related layers (FC). The layer of first convolutional i.e., conv1 presented to close by response institutionalization, with part measure 11, which depicts that each unit in every segment guide is related with $11 \mathrm{X} 11$ neighborhood in the data and stroll of 4, which suggests after every four pixels play out the convolution on the data pictures. The yield of the essential convolution layer is 96 incorporate graphs. The chief layer in instance cov1 slab is trailed by pooling. The piece measure for the pooling is set to 3 with walk 2 . Pooling is trailed by convolution conv 2 with a bit measure 5 as well as walk 2 . This is trailed by totally related layers (FC), for instance, fc3, fc4. In the layer fc4 in AlexNet two exercises are associated. While as in our proposed structure, totally related layer 3 (fc3) is simply presented to revise straight unit action. The yield of our con 4 layer is 256 while in AlexNet 384 part maps are created.

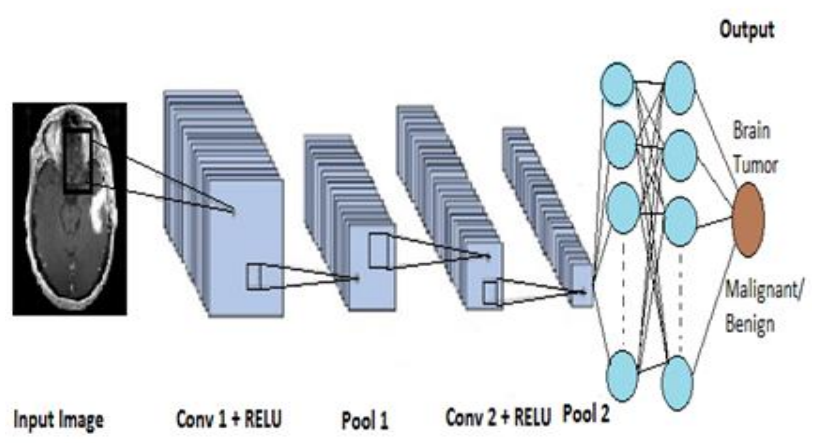

FC3 3 FC4

Figure 1: Proposed CNN architecture

\section{EXPERIMENTAL RESULTS}

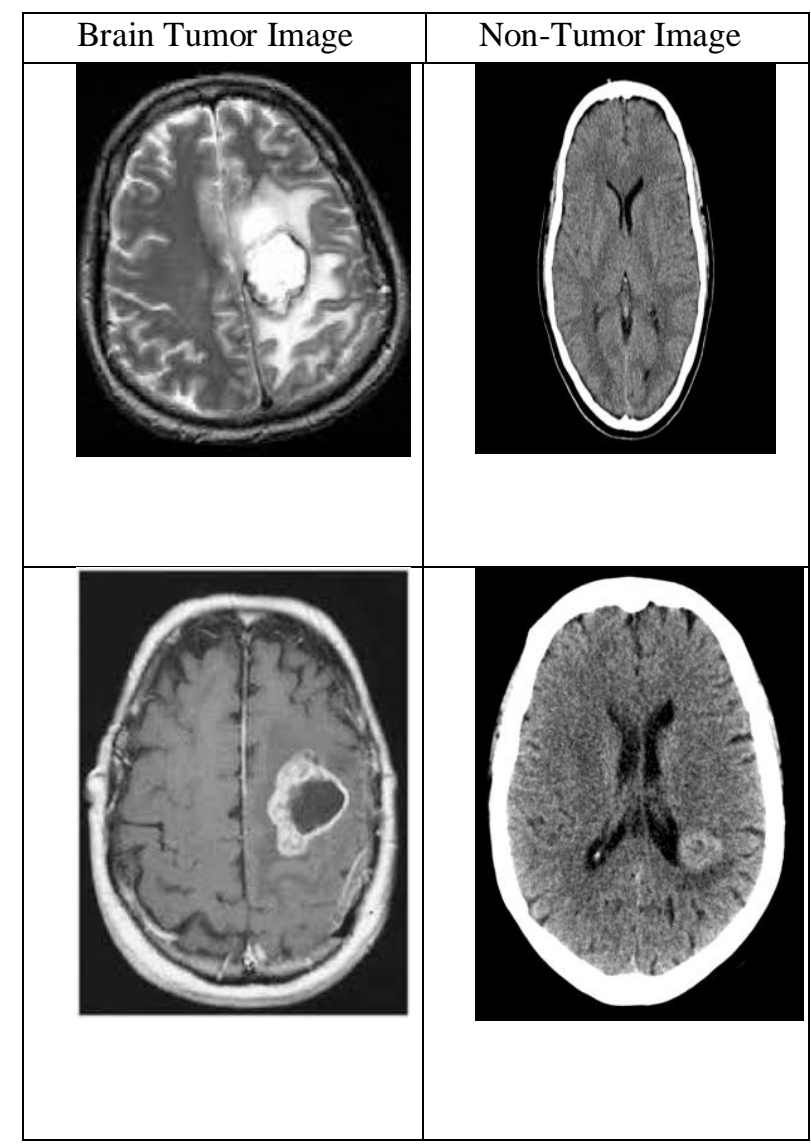

Figure 2: CNN results 


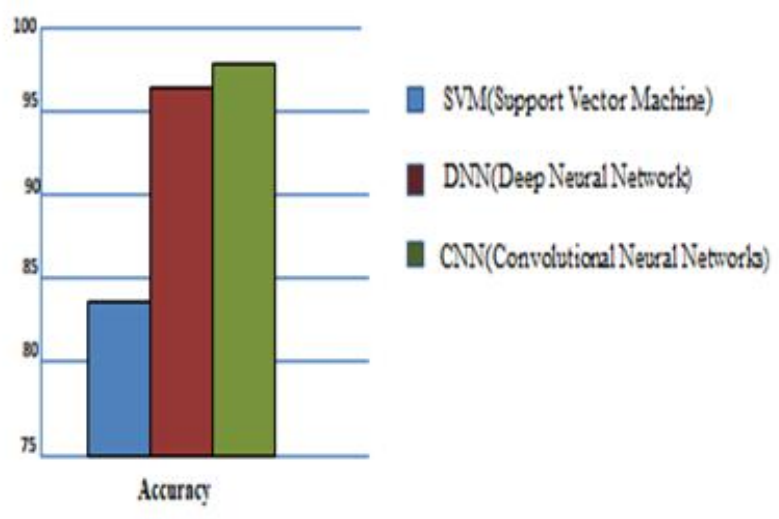

Figure 3: Accuracy of Brain Tumor

The typical brain image has the most minimal likelihood score. Tumor brain has the most elevated likelihood score esteem when contrasted with typical and tumor brain.

\section{CONCLUSIONS}

This paper proposed a changed CNN design that unites various convolution and pooling layers for a progressively raised sum that incorporates learning. The preliminaries for helpful picture life frameworks portrayal have been done and it shows that the proposed CNN incorporates depiction beats the three example models for organizing restorative picture life structures. The change of CNN has been done dependent on experimentation that is finished with the three accomplishment plans. These models overfit as a result of the number of layers and the hyper-parameters used in these plans have been used for the far-reaching course of action of ordinary pictures. In any case, helpful picture informational collections are various to the extent of their obtainment medium and less availability because of the assurance and security approaches when appeared differently with trademark pictures.

In this paper, we furthermore give information into the significant features that have been learned through setting up that will help in examining the various impressions of features stretching out from low measurement to unusual state and their activity uncertain course of action. Our future work will connect with affirmation and request of over the top structures from these assembled life frameworks, inciting a robotized therapeutic picture game plan system.

\section{REFERENCES}

1. Wang, G., Li, W., Maria, A., Zuluaga, Pratt, R, Premal, A., Patel, Aertsen, M., Doel, T., Anna. L. Jan., D., Ourselin, S. \& Vercauteren, T. "Interactive medical image segmentation using deep learning with image-specific fine-tuning”. IEEE Transactions on Medical Imaging, $1-12,2018$.

2. H.-I. Suk, G. Wu and D. Shen "The medical image analysis of deep learning". Annual reviews, 221-248,2017.

3. Sutskever .I, Krizhevsky.A and G. E. n "ImageNet classification with deep convolutional neural networks". In Advances in neural information processing systems, pages 1097-1105, 2012.

4. Williams R. J., Hinton G. E. and D. E. Rumelhart "Learning representations by back-propagating errors". Nature, vol. 323, pp. 533536, Oct. 1986.

5. Y.LeCun et al. "Back-propagation applied to handwritten zip code recognition". Neural Compute, vol. 1, no. 4, pp. 541551, 1989.

6. Miyake .S and Fukushima .K "Neocognitron: A self-organizing neural network model for a mechanism of visual pattern recognition".
In Competition and Cooperation in Neural Nets. Berlin, Germany: Springer, 1982, pp. 267285.

7. T. N. Wiesel and D. H. Hubel "Receptive fields, binocular interaction and functional architecture in the cat's visual cortex". J. Physiol., vol. 160, no. 1, pp. 106154, 1962

8. Rosenblatt .F "The perceptron: A probabilistic model for information storage and organization in the brain". Psychol. Rev., vol. 65, no. 6, pp. 365386, 1958.

9. Pitts .W. and McCulloch W. S. "A nervous activity in ideas of immanent in logical calculus". dlsi.ua.es in the year 1943.

10. G. Litjens et al. (June 2017) "A survey on deep learning in medical image analysis". [Online]Psychol. Rev., vol. 65, no. 6, pp. 365386 , 1958.

11. Shortliffe E.H "Computer-Based Medical Consultations: MYCIN", 286 pp., references, index, illus. 1976

12. R. Smith-Bindman et al "Use of diagnostic imaging studies and associated radiation exposure for patients enrolled in large integrated health care systems", 19962010 JAMA, vol. 307, no. 22, pp. 24002409, 2012.

13. Geoffrey E Hinton, IlyaSutskever and Alex Krizhevsky "In deep convolutions of neural networks of an ImageNet classification". In Advances in neural information processing systems, pages 10971105, 2012.

14. Patrick Haffner, Leon Bottou and YannLeCun "In the Gradient-based learning to applied the document recognition". Proceedings of the IEEE, 86(11):2278-2324, 1998.

15. Yu. Mei, Lu. Zentai, QianjinFeng, Wuhan Chen and Wei Yang "Content-based retrieval of focal liver lesions using bag-of-visualwords representations of single-and multiphase contrast-enhanced CT images". Journal of digital imaging, 25(6):708-719,2012.

16. AnirvanDutta, NiranjanKhandelwal, Sudipta Mukhopadhyay, and Ashis Kumar Dhara "A combination of shape and texture features for classification of pulmonary nodules in lung CT images". Journal of digital imaging, 29(4):466-475, 2016.

17. G. Hinton, Y. Bengio and Y. LeCun "Deep learning" Nature, vol. 521, no. 7553 , pp. $436-444,2017$

18. C. Traina, A.J.M. Traina, and J.C.Felipe "Retrieval by the content of medical images using texture for tissue identification". IEEE Symp. Computer-Based Med. 175-180, 2003.

19. Y. Cui, X. Gao and C. Qiu, Y. Cai "Medical image retrieval based on the deep convolution network and hash coding". International Congress on Image and Signal Processing, Biomedical Engineering and Informatics, 1-6, 2017.

20. A. Geissbuhler, N. Michaux and H. Müller, D. Bandon "A review of content-based image retrieval systems in medical applications-clinical benefits and future directions". International Journal of Medical Informatics, 73, 1-23, 2004.

21. T. Lim and J. Ker, L. Wang "Evaluation of secure multi-party computation for reuse of distributed electronic health data" produced in the year 2017.

22. J. G. Bellika and K. Y. Yigzaw "Evaluation of secure multi-party computation for reuse of distributed electronic health data" IEEEEMBS International Conference on Biomedical and Health Informatics (BHI), 219-222, 2014

23. N. Singh and S. Singh "Object classification to analyze medical imaging data using deep learning" International Conference on Innovations in information Embedded and Communication Systems (ICIIECS), 1-4, 2018.

24. G. Li. Wang, T. Vercauteren, M.Aertsen, S. Ourselin, T. Doel "The deep learning with an image specific fine-tuning of Interactive medical image segmentation"'. IEEE Transactions on Medical Imaging, 1-12, 2018. 